\title{
Preparation of polysaccharide loaded collagen membrane with anti-oxidative activity
}

\author{
Zibin Shu, Shengli Ding, Xiaohong He, Xuemei Dai, Qian Xiao, Min Yang, Xue Leng, \\ Yanshun $\mathrm{Ma}^{*}$ and Hua Yang* \\ College of Chemistry and Materials Science, Sichuan Normal University, Chengdu, Sichuan 610066, \\ China
}

\begin{abstract}
The scavenging activity of polysaccharides from Lycium barbarum, Lentinus edodes and Ganoderma Lucidum Karst to DPPH free radicals was investigated. It was found that among the three polysaccharides, Lycium barbarum polysaccharides (LBP) exhibits the best scavenging activity. Polysaccharide loaded collagen membranes were prepared by mixing LBP with collagen, starch, glycerol, sodium carboxymethyl cellulose and glutaraldehyde. In vitro drug release from membranes was evaluated. With increasing the immersion time, the release rate first increases and then slows down. Meanwhile, the scavenging activity to DPPH radicals exhibits similar variation, in agreement with a good release effect of the membrane. The optimal formulation of collagen membrane and preparation parameters were obtained considering the overall properties and the scavenging activity to radicals.
\end{abstract}

Keywords: Collagen, lycium barbarum polysaccharides, anti-oxidative, drug release, membrane

\section{Introduction}

In fact, many human diseases and premature aging are closely related to the body's excessive oxygen radicals oxidative damage to normal cells. Antioxidants can eliminate these radicals, reduce or even eliminate such oxidative reactions, thus showing beneficial health care effects such as anti-aging and prevention of diseases. Previous studies have shown that skin photodamage caused by UV is also related to oxidative reactions of active oxygens [1]. Plant Polysaccharides constitute a class of natural antioxidants which present antioxidant, anti-cancer, anti-inflammatory, anti-viral, anti-aging, and immune regulatory properties, with little toxic side effects. Polysaccharides present antioxidant effect of scavenging many active oxygen species. Two scavenging mechanisms have been proposed: 1) oxygen radicals are directly removed by the action of polysaccharides; and 2) by increasing the level of intracellular anti-ROS (reactive oxygen species) enzyme, and removal of accumulated lactate

\footnotetext{
* Address for correspondence: Yanshun Ma, College of Chemistry and Materials Science, Sichuan Normal University, Chengdu, Sichuan 610066, China. Tel.: 0086-028-84760802; Fax: 0086-028-84766101; E-mail: mayanshun05@163.com.

Hua Yang, College of Chemistry and Materials Science, Sichuan Normal University, Chengdu, Sichuan 610066, China. Tel.: 0086-028-84760802; Fax: 0086-028-84766101; E-mail: scsdyh@126.com.
} 
dehydrogenase (LDH) and malondialdehyde (MDA), the anti-oxidation activity of cells is improved, thereby reducing the oxidative damage [2].

Polysaccharides can be extracted from a variety of plants such as Lycium barbarum, Lentinus edodes and Ganoderma Lucidum Karst. These compounds present antioxidant effects by inhibiting production of oxygen radicals, and scavenging hydroxyl radicals $(\mathrm{OH} \bullet)$ and superoxide anion $\left(\mathrm{O}_{2}^{-\bullet}\right)$ [3]. Lycium barbarum is the dry fruit of Lycium barbarum belonging to Solanaceae. Lentinus edodes is the second most cultivated edible mushroom in the world. They are both medicinal and edible substances. Ganoderma lucidum Karst is a basidiomycete belonging to polyporaceae, and used as a medicinal mushroom. All these plants present anti-tumor, anti-aging, antioxidation, and immune regulation functions [4-6].

On the other hand, collagen is a natural polymer with good biocompatibility, and has been widely used cosmetic and biomedical applications [7]. In this work, the scavenging activity to DPPH $\bullet$ radicals of polysaccharides derived from Barbary wolfberry fruit, Lentinus edodes and Ganoderma Lucidum Karst will be evaluated. The polysaccharide with optimal activity will be used to develop a collagen based membrane for preventing skin photo-damage, photo-ageing or skin cancer.

\section{Experimental section}

\subsection{Materials}

Collagen (laboratory prepared); soluble starch (AR); glutaraldehyde (AR); glycerol (AR); sodium carboxymethyl cellulose (CP); absolute ethanol (AR); Lycium barbarum polysaccharide (LBP), lentinan, Ganoderma lucidum polysaccharides(GLP) (Shaanxi Ruikang Biological Engineering Co., Ltd.), 1,1-diphenyl-2-picrylhydrazyl (DPPH) (Tokyo Kasei Kogyo Co., Ltd (TCI)).

\subsection{Methods}

\subsubsection{Preparation of polysaccharide loaded collagen membrane with anti-oxidative activity}

Novel polysaccharide loaded collagen membrane is prepared using homemade collagen[8], starch, sodium carboxymethyl cellulose (CMC-Na) as the membrane material, glutaraldehyde as cross-linking agent, and glycerin as humectant. Plant polysaccharide is added in the mixture during preparation.

The procedure consists in 4 steps:

(1) CMC-Na is added in water with a ratio of $1: 18$. The mixture is stirred to yield a transparent jelly;

(2) $0.5 \mathrm{~g}$ collagen powder is dissolved in $1 \mathrm{~mL}$ water. $0.1 \mathrm{~g}$ starch is then added, followed by stirring at $40^{\circ} \mathrm{C}$ till complete dissolution. $3.0 \mathrm{~g} 50 \%$ glutaraldehyde and 2.5-3.0 g glycerin are successively introduced, and mixed to yield a homogeneous solution;

(3) $10.0 \mathrm{~g}$ aqueous solution of $\mathrm{CMC}-\mathrm{Na}$ is added to the mixture, and homogenized by stirring at $40^{\circ} \mathrm{C}$. 0-2.0g plant polysaccharide is then added, and stirred to yield a paste-like solution;

(4) The paste is spread on a template and freeze dried, yielding a polysaccharide loaded collagen membrane.

\subsubsection{Measurements}

UV measurements of plant polysaccharides and polysaccharide loaded collagen membrane were performed with 2802 UV-VIS spectrophotometer (Shanghai UNICO). 


\section{Results and discussion}

\subsection{Comparison of the activity of plant polysaccharides}

DPPH is usually used as a substrate to evaluate antioxidative activity of antioxidants [9]. The antioxidant activity of three plant polysaccharides, i.e. LBP, lentinan and GLP was evaluated using DPPH method. The polysaccharide with optimal activity was selected for the preparation of polysaccharide loaded collagen membrane.

DPPH is a stable radical with a strong absorption at a wavelength of $517 \mathrm{~nm}$. The absorption will weaken when radical scavengers make pair with DPPH in solution. The decrease of absorption is proportional to the quantity of paired radicals. Therefore, the change in absorbance can be used to assess the scavenging activity of antioxidants to radicals [10]. Radical scavenging activity is expressed with scavenging ratio, and the higher the scavenging ratio, the stronger the antioxidation capacity. The scavenging ratio was measured following the method of Huang et al. with modifications [11].

Plant polysaccharides (LBP, lentinan and GLP) were dissolved in water at concentrations of 1.0, 2.0, $3.0,4.0,5.0,6.0,8.0$ and $10.0 \mathrm{mg} / \mathrm{mL}$.

DPPH radicals were dissolved in ethanol to yield a $2 \times 10^{-4} \mathrm{M}$ solution.

$2 \mathrm{~mL}$ sample solution was mixed with $2 \mathrm{~mL}$ DPPH solution in a test tube, and homogenized. The absorption of the solution was measured at $517 \mathrm{~nm} 30 \mathrm{~min}$ after mixing to give a value $\mathrm{A}_{\mathrm{i}} .2 \mathrm{~mL}$ sample solution was mixed with $2 \mathrm{~mL}$ ethanol and the absorbance value was denoted as $\mathrm{A}_{\mathrm{j}} .2 \mathrm{~mL}$ of water was mixed with $2 \mathrm{~mL}$ DPPH solution and the absorbance value was denoted as Ac.

The scavenging ratio was determined using the following equation:

$$
\text { Scavenging ratio }(\%)=\left[1-\left(\mathrm{A}_{\mathrm{i}}-\mathrm{A}_{\mathrm{j}}\right) / \mathrm{A}_{\mathrm{c}}\right] \times 100 \%
$$

The scavenging ratio was plotted against the concentration of plant polysaccharide as shown in Figure 1.

With increasing polysaccharide concentration, the scavenging ratio rapidly increases. Beyond 6 $\mathrm{mg} / \mathrm{mL}$, the increase slows down. LBP exhibits the highest scavenging ratio in the whole concentrations range. In the cases of GLP and lentinan, the former shows better scavenging ratio in the low concentration range, but lower scavenging ratio in the high concentration range than the latter. The concentration of plant polysaccharides to reach $50 \%$ scavenging ratio (IC50) is 1.9, 4.0 and 4.2 $\mathrm{mg} / \mathrm{mL}$ for LBP, GLP and lentinan, respectively. It is preferable that polysaccharide presents good anti-oxidation activity in a wide range of concentrations since its concentration will gradually decrease due to sustained release from polysaccharide loaded membranes. Therefore, LBP was selected as an active component for the preparation of polysaccharide loaded collagen membrane with anti-oxidative activity.

\subsection{Determination of the amount of Lycium barbarum polysaccharides}

Polysaccharide loaded collagen membrane were prepared according to the above procedure with various amounts of LBP. The properties of membranes were evaluated using single factor method, as shown in Table 1.

Table 1 shows that when the amount of LBP is too high, the membranes exhibit poor ductility and flexibility. The membrane is water soluble in the absence of polysaccharide. However, with increase of the amount of polysaccharide, the water solubility becomes poorer and the membranes gradually swell in water at $37^{\circ} \mathrm{C}$. This finding indicates the occurrence of inter-molecular interactions between 
Table 1

Influence of the amount of LBP on the membrane properties

\begin{tabular}{|c|c|c|c|c|c|c|c|c|}
\hline No & 1 & 2 & 3 & 4 & 5 & 6 & 7 & 8 \\
\hline CMC-Na solution (g) & 10.0 & 10.0 & 10.0 & 10.0 & 10.0 & 10.0 & 10.0 & 10.0 \\
\hline Starch $(g)$ & 0.1 & 0.1 & 0.1 & 0.1 & 0.1 & 0.1 & 0.1 & 0.1 \\
\hline Glutaraldehyde (g) & 3.0 & 3.0 & 3.0 & 3.0 & 3.0 & 3.0 & 3.0 & 3.0 \\
\hline Glycerin $(\mathrm{g})$ & 2.5 & 2.5 & 2.5 & 2.5 & 2.5 & 2.5 & 2.5 & 2.5 \\
\hline Collagen protein $(\mathrm{g})$ & 0.5 & 0.5 & 0.5 & 0.5 & 0.5 & 0.5 & 0.5 & 0.5 \\
\hline $\operatorname{LBP}(\mathrm{g})$ & 0 & 0.1 & 0.3 & 0.5 & 0.8 & 1.0 & 1.5 & 2.0 \\
\hline Ductility of membrane & Very good & Very good & & & & & poor & poor \\
\hline $\begin{array}{l}\text { Flexibility } \\
\text { Water solubility* }\end{array}$ & $\begin{array}{l}\text { Very good } \\
\text { soluble }\end{array}$ & Very good & good & & $\rightarrow$ & swollen & $\underset{\text { swollen }}{\longrightarrow}$ & $\begin{array}{l}\text { poor } \\
\text { swollen }\end{array}$ \\
\hline
\end{tabular}

Note: The arrow indicates a gradual change

polysaccharide and the membrane matrix.

Considering the overall properties of membranes, $0.1 \sim 0.8 \mathrm{~g}$ polysaccharide appears most appropriate for membrane preparation.

\subsection{In vitro release of polysaccharide from membranes}

\subsubsection{UV absorption of Lycium barbarum polysaccharide}

The UV absorption of polysaccharide solution is measured at $0.3 \mathrm{mg} / \mathrm{mL}$. As shown in Figure 2, LBP presents a maximum absorption peak at $200 \mathrm{~nm}$, and a smaller peak at $284 \mathrm{~nm}$. The absorption becomes very weak beyond $350 \mathrm{~nm}$. Thus LBP has an absorption in the range of 200-350 nm.

\subsubsection{Determination of polysaccharide release from collagen membrane}

In vitro release of polysaccharide from collagen membrane was evaluated from two aspects.

(1) Relationship between the immersion time and the concentration of released drug

A membrane loaded with $0.3 \mathrm{~g}$ of polysaccharide was soaked in $100 \mathrm{~mL}$ of distilled water. After 0.5 , $1,2,4,6,8,12$ and $24 \mathrm{~h}, 2 \mathrm{~mL}$ of the supernatant was taken and diluted with $2 \mathrm{~mL}$ water. The UV absorption spectra of the various solutions are shown in Figure 3.

Figure 3 shows that, the maximum absorption of seven curves sequentially shifts from $200 \mathrm{~nm}$ to $220 \mathrm{~nm}$. Beyond $350 \mathrm{~nm}$ the absorption becomes very weak. Thus the UV absorption of the release

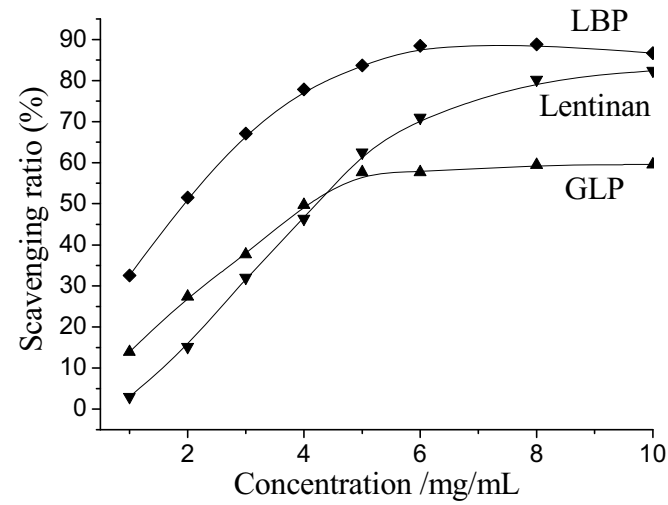

Fig. 1. Scavenging ratio changes as a function of the concentration of plant polysaccharide.

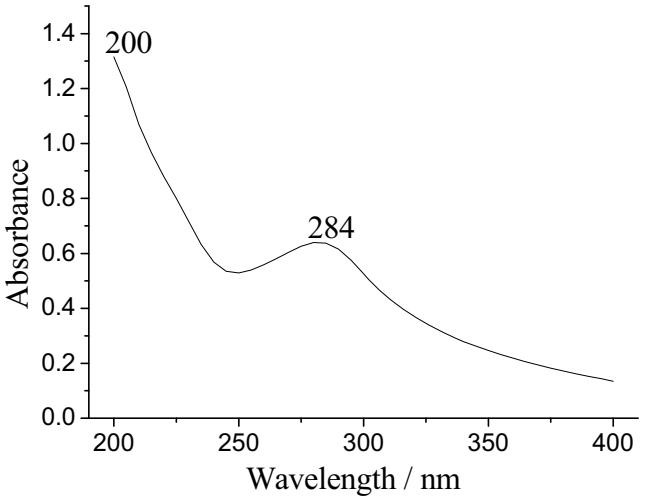

Fig. 2. UV absorption spectrum of LBP solution. 
medium is in the range $200-350 \mathrm{~nm}$, which well agrees with the absorption range of LBP. It is thus concluded that higher absorption of the release medium indicates higher polysaccharide concentration. Moreover, with increasing immersion time, the UV absorption initially exhibits a sharp increase, followed by a slower increase. This finding indicates a sustained release of polysaccharide from the membrane.

(2) Determination of the scavenging ratio of collagen membrane to DPPH

Collagen membranes loaded with $0,0.2,0.3$, and $0.8 \mathrm{~g}$ of polysaccharide were prepared, and soaked in $100 \mathrm{~mL}$ distilled water. After 2, 4, 6, 8, 12 and $24 \mathrm{~h}$ immersion, $2 \mathrm{~mL}$ of the supernatant was taken, and the scavenging ratio to DPPH radicals was measured. The results are shown in Figure 4.

Figure 4 shows that the scavenging ratio of membranes is rather low in the absence of polysaccharide. After $24 \mathrm{~h}$, the scavenging ratio only reaches $6.7 \%$. The addition of LBP in the membranes results in increase of the scavenging ratio. The higher the amount of loaded polysaccharide, the higher the scavenging ratio. On the other hand, the scavenging ratio rapidly increases in the first 6 hours of immersion, reaching $63-70 \%$ of the total ratio obtained at $24 \mathrm{~h}$. Beyond, the increase slows down. This is because the concentration of polysaccharide in the membrane is initially high, and the release is fast, thus leading to fast increase of the scavenging ratio. With the increase of immersion time, the release rate becomes slower since the concentration of remaining polysaccharide is decreasing. After $24 \mathrm{~h}$, the scavenging ratio reaches 9,18 and $28 \%$ for collagen membranes loaded with $0.2,0.3$ and $0.8 \mathrm{~g}$ of polysaccharide, respectively. The scavenging ratio is rather low with $0.1-0.2 \mathrm{~g}$ loaded LBP. Therefore, the optimal range of loaded LBP is $0.3-0.8 \mathrm{~g}$. It should be noted that the scavenging ratios shown in Figure 4 are rather low because they were obtained at low concentrations of polysaccharide in the immersion medium. The scavenging ratio should be much higher in practical uses since the concentration of released LBP would be much higher due to small amount of surface fluid. Furthermore the interaction between released LBP and oxygen radicals will enhance the release of LBP, thus improving the scavenging activity.

The results in Figures 3 and 4 indicate that the novel polysaccharide loaded collagen membrane exhibits a sustained release behavior of polysaccharide in vitro.

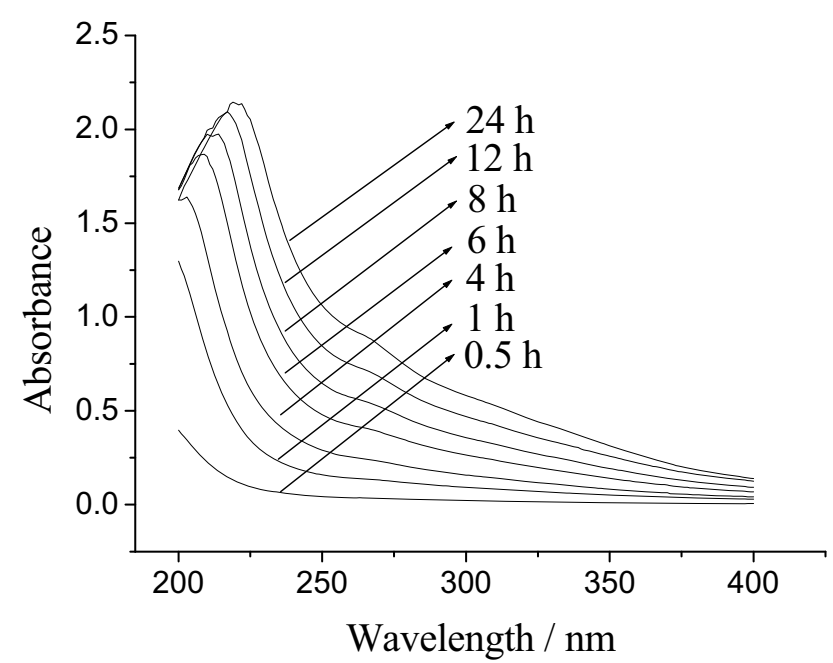

Fig. 3. UV absorption spectra of solutions after different soaking times.

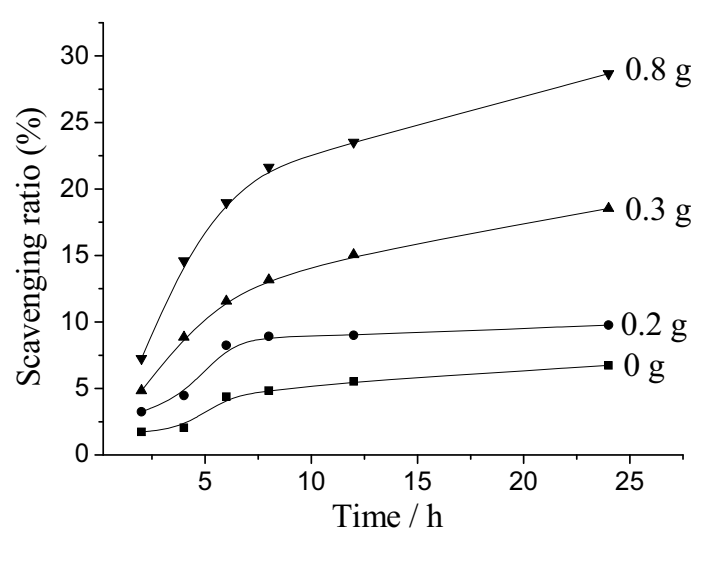

Fig. 4. Scavenging ratio changes as a function of soaking time of collagen membranes loaded with various amounts of polysaccharide. 


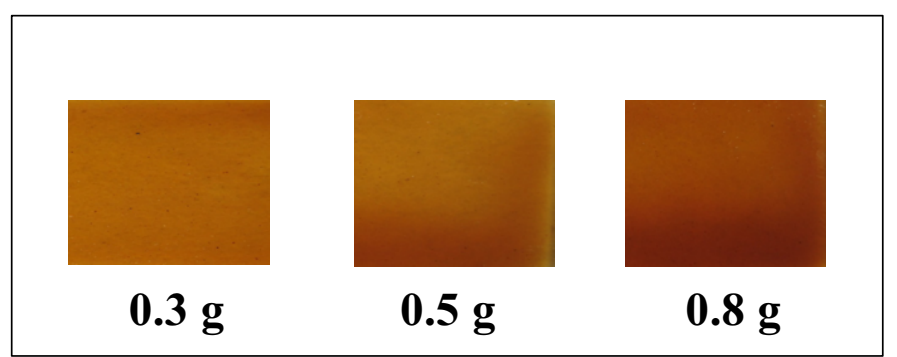

Fig. 5. Images of collagen membranes with different LBP loads.

\subsection{Homogeneity of samples}

The homogeneity of samples was evaluated by visual examination. As shown in Figure 5, the color of membranes gradually changes from light brown to dark brown with increasing amount of LBP up to $0.8 \mathrm{~g}$. The membranes appear smooth at the surface. The distribution of polysaccharide with the matrix is homogeneous in a wide range of concentrations. Polysaccharide can hardly aggregate due to the viscosity of the paste before drying, thus it is evenly dispersed in the dried membrane.

The optimal procedure of membrane preparation is described as follows: $0.5 \mathrm{~g}$ collagen, $0.1 \mathrm{~g}$ starch, $3.0 \mathrm{~g}$ glycerol, $3.0 \mathrm{~g}$ glutaraldehyde, and $10.0 \mathrm{~g}$ sodium carboxymethyl cellulose solution are mixed at $40^{\circ} \mathrm{C}$ to yield a thick paste. $0.3-0.8 \mathrm{~g}$ LBP is then added. After stirring, the mixture is evenly spread on a glass template and freeze dried to yield a polysaccharide-collagen membrane with anti-oxidative activity.

\section{Conclusion}

This work studied the scavenging activity of LBP, lentinan and Ganoderma lucidum polysaccharides to $-\mathrm{DPPH}$ radicals. LBP polysaccharide exhibited the best scavenging activity and was selected to prepare novel collagen based membranes with anti-oxidative activity. LBP polysaccharide was mixed with homemade collagen, starch, sodium carboxymethyl cellulose solution, glycerol and glutaraldehyde. The resulting membranes present good homogeneity, ductility and flexibility in a wide range of polysaccharide contents. In vitro release experiments showed that with increasing soaking time in water, the released drug concentration and the scavenging ratio to $-\mathrm{DPPH}$ radicals rapidly increase at the beginning, and then the increase slows down. This finding revealed that the membranes exhibit a sustained release effect due to the strong interaction between the drug and the membrane matrix.

The novel collagen membrane presents good biocompatibility, cell adaptability, as well as antibacterial, anti-oxidation, anti-cell aging, and long-acting release properties, providing a new type of biomaterials for preventing light skin aging, skin cancer and other light damages. Further studies are underway to evaluate the practical applications of membranes.

\section{Acknowledgments}

The authors thank the Ministry of Education (No. 201410636007) and the Education Department of Sichuan Province (No. 201410636036) for financial support. 


\section{References}

[1] F. Afaq, V.M. Adhami and H. Mukhtar, Photochemoprevention of ultraviolet B signaling and photocarcinogenesis, Mutation Research 571 (2005), 153-173.

[2] Z.H. Zhao, X.W. Zheng and F. Fang, Ganoderma lucidum polysaccharides supplementation attenuates exercise-induced oxidative stress in skeletal muscle of mice, Saudi Journal of Biological Sciences 21 (2014), 119-123.

[3] J.L. Zhang, H.X. Wang, W. Zhang and X.Y. Zhang, Anti-oxidation activity of ganodorma lucidum and lycium chinensis polysaccharides compounds, Food and Machiney 20 (2004), 11-46.

[4] L.L. Zhang, J.X. Gu, YJ. Chen and L. Zhang, A study on four antioxidation effects of lycium barbarum polysaccharides in vitro, African Journal of Traditional, Complementary, and Alternative Medicines 10 (2013), 494-498.

[5] Y.Y. Zhang, S. Li, X.H. Wang, L.N. Zhang and P.C.K. Cheung, Advances in lentinan: Isolation, structure, chain conformation and bioactivities, Food Hydrocolloids 25 (2011), 196-206.

[6] Z.B. Lin and H.N. Zhang, Anti-tumor and immunoregulatory activities of ganoderma lucidum and its possible mechanisms, Acta Pharmacologica Sinica 25 (2004), 1387-1395.

[7] B. Wang, D.Y. Jia, S.Q. Ruan and S. Qin, Structure and properties of collagen-konjac glucomannan-sodium alginate blend films, Journal of Applied Polymer Science 106 (2007), 327-332.

[8] H. Yang, X.X. Chen, Z.B. Shu, X.F. Guo, R.H. Gao, S.Y. Zhou and Y.C. Yan, Preparation of collagen wound-healing membranes, Journal of Chemical and Pharmaceutical Research 5 (2013), 655-658.

[9] X.M. Li, X.L. Li and A.G. Zhou, Evaluation of antioxidant activity of the polysaccharides extracted from lycium barbarum fruits in vitro, European Polymer Journal 43 (2007), 488-497.

[10] M.S. Blois, Antioxidant determinations by the use of a stable free radical, Nature 181 (1958), 1199-1200.

[11]X.Q, Huang, Z.C. Tu, Y. Jiang, H. Xiao, Q.T. Zhang and H. Wang. Dynamic high pressure microfluidization-assisted extraction and antioxidant activities of lentinan, International Journal of Biological Macromolecules 51 (2012), 926-932. 